U.S. Department of Energy

Office of Science and Office of Environmental Management

Environmental Management Science Programs

Office of Science Project 86952

\title{
MULTI-REGION REACTIVE TRANSPORT DUE TO STRONG ANISOTROPY IN UNSATURATED SOILS WITH EVOLVING SCALES OF HETEROGENEITY
}

\section{FY 2005 ANNUAL REPORT}

Lead Institutions: Pacific Northwest National Laboratory

Support Institution: Lehigh University

Principal Investigator: Dr. Andy Ward

Phone: (509) 372-6114

FAX: (509) 372-6089

Email: andy.ward@pnl.gov

Pacific Northwest National Laboratory

P.O. Box 999, MS K9-33

Battelle Blvd, Richland, WA 99352

Co-Investigator: Dr. Glendon Gee

Phone: (509) 372-6096

FAX: (509) 372-6089

Email: glendon.gee@pnl.gov

Pacific Northwest National Laboratory

P.O. Box 999, MS K9-33

Battelle Blvd, Richland, WA 99352

Co-Investigator: Dr. Mark White

Phone: (509) 372-6070

FAX: (509) 372-6089

Email: mark.white@pnl.gov

Pacific Northwest National Laboratory

P.O. Box 999, MS K9-33

Battelle Blvd, Richland, WA 99352
Co-Principal Investigator: Dr. Horace Moo-Young

Phone: (610) 758-6851

FAX: (610) 758-6405

Email: hkm3@lehigh.edu

Depart. of Civil and Environmental Engineering

Lehigh University

13 E. Packer Ave., Bethlehem, PA 18015

Co-Investigator: Dr. Dave Rector

Phone: (509) 372-4530

FAX: (509) 375-3641

Email: david.rector@pnl.gov

Pacific Northwest National Laboratory

P.O. Box 999, MS K7-15

Battelle Blvd, Richland, WA 99352

Co-Investigator: Steve Yabusaki

Phone: (509) 372-6095

FAX: (509) 372-6089

Email: yabusaki@pnl.gov

Pacific Northwest National Laboratory

P.O. Box 999, MS K9-33

Battelle Blvd, Richland, WA 99352 


\subsection{Background}

At Hanford, the prediction of field-scale flow and transport in the vadose zone beneath tank farms and other waste-management facilities provide as good example of the limitations of current conceptualizations. Contaminant plumes in Hanford's vadose zone typically show extensive lateral spreading with splitting along flow paths and multiple zones of high-contaminant concentrations, even in sediments that appear homogeneous and isotropic at the regional scale. Because of the limited success in predicting current contaminant distributions using existing conceptual models and approaches to parameterization, there is some uncertainty about predictions of future transport behavior. This is mostly because current parameter upscaling procedures result in overly smoothed descriptions of the hydraulic functions that cause many of the important details (e.g. extreme water and solute flux, anisotropy), known to be caused by finescale heterogeneity, to be ignored.

\subsection{Research Hypotheses and Objectives}

The preferential alignment of particles during sedimentation results in a microscale pore structure that renders the porous medium anisotropic in their resistance to the flow of mass and energy at the pore scale. The net effect of the pore-scale structure coupled with macroscale layering and heterogeneity is directionally dependent correlation lengths and anisotropic intrinsic properties

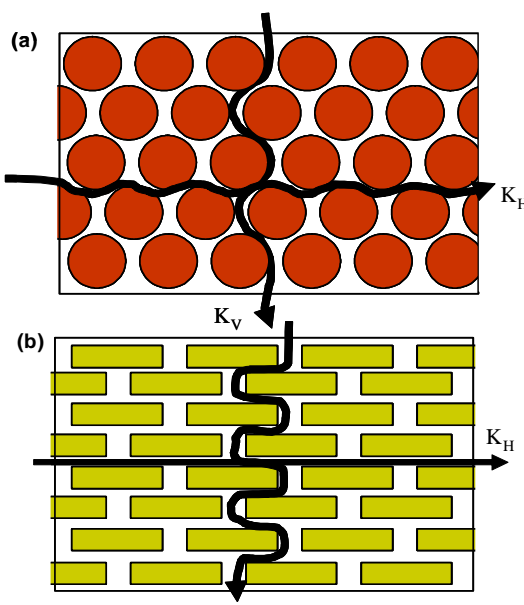

Figure 1. Conceptualization of Porescale Anisotropy, (a) spherical particles (e.g. sand) (b) flat particles (e.g. mica)
Soils formed from the deposition of spherical or rounded particles, such as sands, would tend show similar pore connectivity, tortuosity, permeability and dispersivity in all directions, i.e. they would tend to be isotropic at the pore scale (Figure 1a). In contrast, soils formed from the settling of flat particles and rods, which settle with their long axes horizontal, would tend to be anisotropic (Figure 1b). Because of the higher degree of tortuosity and smaller degree of connectivity normal to the long axes, one might expect the vertical conductivity, $\mathrm{K}_{\mathrm{V}}$ to be less than that parallel to the long axes, i.e. the horizontal hydraulic conductivity, $\mathrm{K}_{\mathrm{H}}$. The scope was to develop a rigorous mathematical model for describing saturation dependent anisotropy in natural sediments, most of which are not amenable to description by the Yeh (1985) model.

Specific objectives included:

1. Quantify the effect of pore-scale heterogeneity on intrinsic hydraulic properties and derive the mechanistic relationship to particle and pore size statistics

2. Determine the scale of applicability and the limitations of the small perturbation (stochastic) approach for upscaling flow parameters in heterogeneous anisotropic porous media

3. Develop an appropriately rigorous averaging approach to better quantify macroscale flow behavior based on pore-scale heterogeneity 
These objectives are being met through an integrated experimental and modeling study that encompasses three scale- pore-scale (Lattice Boltzman, LB; Smooth particle hydrodynamics, $\mathrm{SPH}$ ); centrifuge measurements using the unsaturated flow apparatus (UFA); core scale; and macroscopic scale.

At the pore scale, experimental and modeling efforts have focused on characterization of pore morphology and quantifying the effects of pore-scale heterogeneities on flow using pore-scale models (Lattice Boltzmann and Smooth Particle Hydrodynamics). Small column (5 cm cross section) measurements have made in the unsaturated flow apparatus (UFA lab centrifuge system) using repacked and undisturbed cubed-shaped sediment samples to obtain flow and transport data to validate the pore-scale models. Mesoscale experiments (15 cm cross section) have also been used in a three-axis permeameter to measure effective hydraulic properties and their directional dependence. All of the tests have been repeated in a larger three-axis permeameter (30 -50 cm cross section) on geocentrifuge to quantify parameters at a larger scale using the same sediments and packing configurations.

\subsection{Research Progress and Implications}

\subsection{Pore-scale Anisotropy}

Owing to the difficulty in sampling and interrogating the unconsolidated sediments typical of Hanford, a physically based sedimentation model was developed to simulate different packing arrangements of particles ranging from spheres to disks. To gain a quantitative understanding of the combined effects of three dimensional particle arrangement and particle shape on the permeability tensor, flow through the different packs was predicted using a lattice-Boltzmann (LB) flow simulator (Stewart et al, 2004; 2005). Figure 2 shows a random pack of oblate

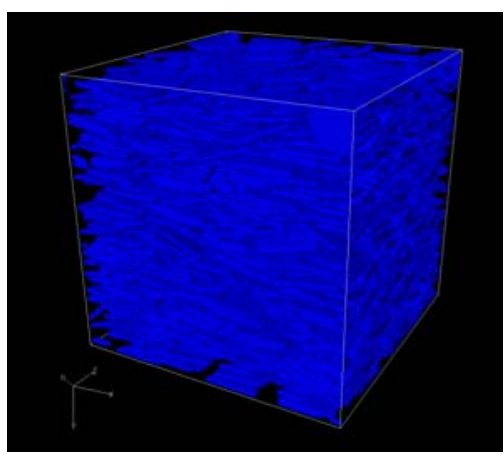

Figure 2: Random pack of oblate ellipsoids with a 10:1 aspect ratio.

ellipsoids with an aspect ratio of 10:1. Results show that the intrinsic properties of the porous medium and the degree of anisotropy in depends not only upon particle shape and alignment, but also on the three dimensional structure of the pack (Stewart et al, 2005; Ward, 2005). With respect to permeability, trends for random packs indicate that more oblate particles and higher degrees of particle alignment lead to decreased pore connectivity and increased tortuosity perpendicular to the direction of maximum alignment, which results in greater anisotropy.

Figure 3 shows the corresponding anisotropy ratios, $\mathrm{A}_{\mathrm{xz}}=\mathrm{k}_{\mathrm{x}} / \mathrm{k}_{\mathrm{z}}$ for body centered cubic (bcc) and face centered cubic (fcc) configurations. As expected, $\mathrm{A}_{\mathrm{xz}}=1$ for spherical particles $\left(\mathrm{r}_{\mathrm{X}} / \mathrm{r}_{\mathrm{Z}}=1\right)$, a result consistent with field measurements and continuum scale numerical simulations in coarse sand. $A_{x z}$ ranged from 1 to 14 in the fcc packs and were significantly higher than in the bcc packs increasing as aspect ratio increased. In contrast, $A_{x z}$ in the bcc packs decreased from a maximum of 1 to 0.12 as aspect ratio increased from 1 to 5 . The dependence of permeability on

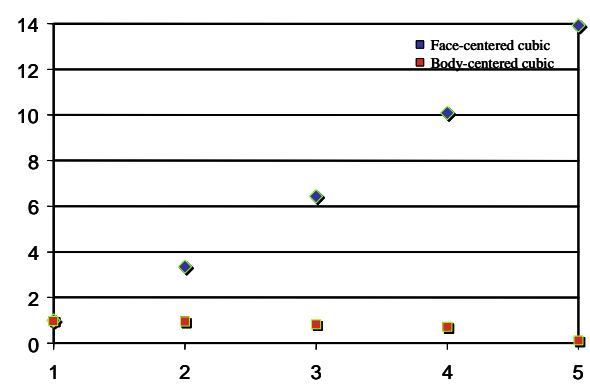

Figure 3: $A_{x z}$ for the dimensionless $k$ in packs of oblate ellipsoids with varying aspect ratios. aspect ratio is consistent with our underlying hypothesis that particle shape and arrangement would impact pore scale anisotropy, but the different trends were somewhat counter-intuitive. 
Nevertheless, the increase in $A_{x z}$ with increasing aspect ratio is more likely to occur in field soils since the fcc arrangement, being the most stable, is qualitatively more similar to natural packs. As the bed is stretched in the horizontal directions, the longer pores lead to significantly greater permeabilities along the $x$ and $y$ axes, and therefore higher anisotropy ratios. We have since extended the LB simulator to handle multiphase simulations using a novel approach to overcome the viscosity and density ratio limitations that affect unsaturated flow simulations. We are also using the SPH model, which does not have any stability problems due to viscosity and density, to predict macroscopic properties from the pore scale simulations.

The particle packing algorithm, PacSIM, developed as part of this project was selected for further development under the LDRD program this FY. The is already being used to support several independently funded projects including 1) simulation of ceramic filtration media directly funded by the DOE Office of Freedom Car and Vehicle Technology, and also by a cooperative research agreement (CRADA) with Dow Automotive, and 2) 1831 project involving beds of irregular particles in an advanced chemical reactor. Figure 4 shows an example of a simulation of catalyst pellets packed into an experimental reactor with streamlines and high velocity regions.

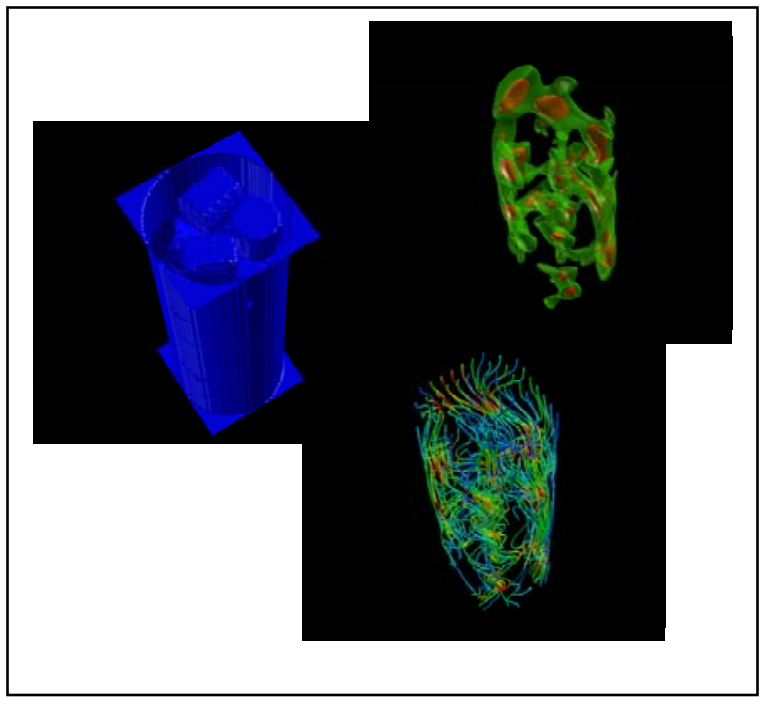

Figure 4. Simulation of catalyst pellets packed into an experimental reactor with streamlines and high velocity regions

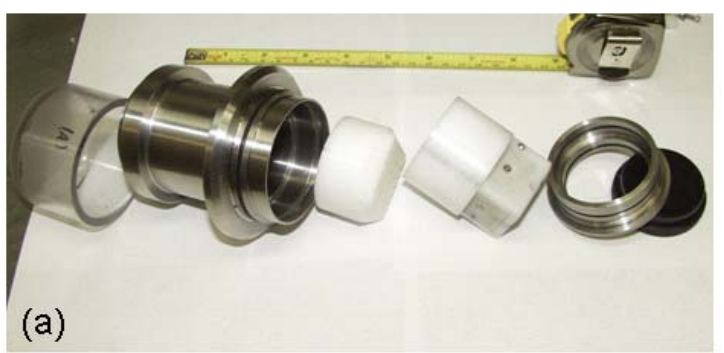

\subsection{Pore-scale Anisotropy}

A special cubic cell was developed for use in the unsaturated flow apparatus (UFA) to allow measurement of directional unsaturated hydraulic properties and evaluation of the pore connectivity tensor concept. This cell allows us to subsample undisturbed field cores collected in lexan liners 4" in diameter or larger. This cell was also instrumented to allow directional measurement of electrical conductivity. Directional measurements of electrical and hydraulic properties are now routinely made in the UFA for a variety of sediments. Figure 5 shows the components of UFA sample holder (Figure 5a) and soil cube sample holder (Figure 5b) used to make directional measurements of water content, permeability, and electrical conductivity as functions of saturation in unconsolidated soils.

(b)

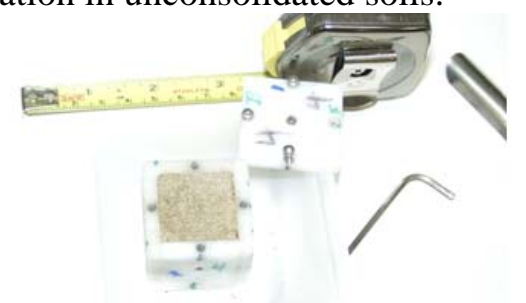

Figure 5. Sample holder and components form directional permeability measurements using the UFA, (a) UFA sample holder, (b) cube insert for UFA sample holder. 
The influence of saturation on $K(h)$ is illustrated in Figure 5 for four sediments with increasing heterogeneity (via aspect ratio) and therefore increasing pore connectivity in the horizontal direction. The solid lines are the best-fits using the new anisotropy model that treats pore connectivity as a tensor. As expected spherical particles (Fig. $6 a)$ gave rise to an isotropic $K(h)$. As the aspect ratio increases, so does the degree of anisotropy (Figure 6b,c,d). Existing models ignore this effect. This model has been for saturation dependent anisotropy overcomes the limitations of the widely used stochastic models that are based on small perturbation solutions to the Richards'

flow equation. The stochastic approach is based on the assumption of a exponential relationship between $K$ and $h$, a relationship that has been shown to be of
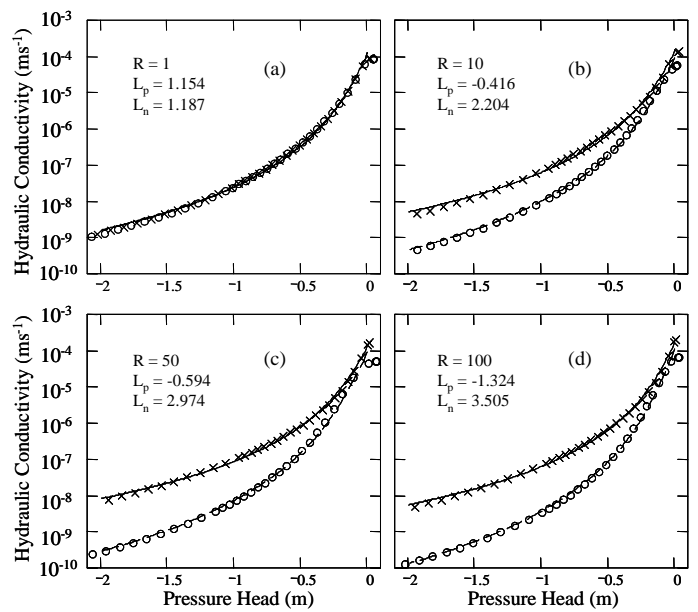

Figure 6: $K(h)$ showing effects of increasing pore connectivity ( $a=$ spherical particles ). limited use with Hanford sediments. With the new approached developed as part of this research, we can use any of the widely used water retention and hydraulic conductivity functions (BrooksCorey, van Genuchten) that are based on the statistical pore-size distribution concept. This approach has already been incorporated into the PNNL-developed STOMP simulator and is being applied to solving flow and transport problems on site.

\subsection{Macro-scale Anisotropy}

The tortuosity-connectivity tensor that formed the basis of our original hypothesis and confirmed with pore scale modeling has been tested at the macroscopic scale using measurements made on undisturbed and repacked sediments in a geocentrifuge. The pore tortuosityconnectivity tensor concept has been applied to the continuum scale unsaturated hydraulic conductivity tensor $\boldsymbol{K}(S)$ for anisotropic soils. Definition of $\boldsymbol{K}(S)$ requires three tortuosityconnectivity coefficients, one for each principal direction. This definition implies saturationdependent anisotropy and is applicable to all models of relative permeability that assume a statistical pore-size distribution. Anisotropy is invoked simply by incorporating a connectivity tensor, $\mathbf{L}_{\mathrm{i}}$. This approach is superior to existing saturation dependent anisotropy models (e.g. Yeh 1985). The model can be used with any type of hydraulic function without the need simplifying assumptions about linearity parameterization is straightforward. It also overcomes current limitations that require $\mathrm{K}_{\mathrm{x}}>\mathrm{K}_{\mathrm{z}}$; the model can describe anisotropy equally well for $\mathrm{K}_{\mathrm{x}}<\mathrm{K}_{\mathrm{z}}$ and $\mathrm{K}_{\mathrm{x}}>\mathrm{K}_{\mathrm{z}}$ (Figure 7).

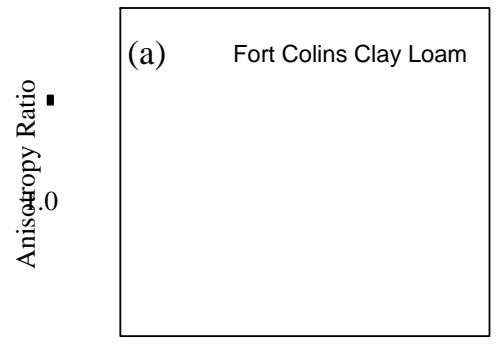

Pressure Head $(\mathrm{cm})$

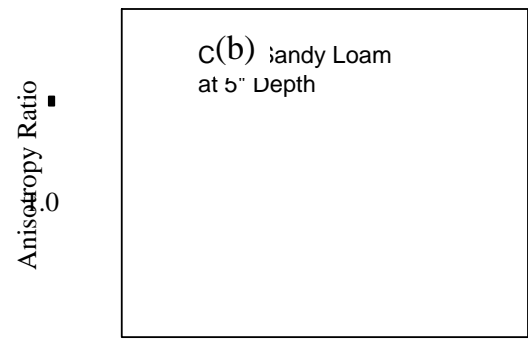

Pressure Head (cm)

Figure 7. Measured and predicted anisotropy in $K(h)$ for (a) clay loam with $K_{x}>K_{z}, L_{x}=-1.08$ (b) sandy loam with $K_{x}<K_{z} ; L_{x}=2.37$. 
The implications of this research are quite significant. Lateral flow due to variable anisotropy has dominated every field experiment (both planned and unplanned) performed at the Hanford Site, it is inadequately described in all but a few existing models of unsaturated flow. Those models that do consider lateral flow are formulated on the presumption that flow can be linearized and treated as a small perturbation to the unsaturated flow dynamics and, therefore, are in contradiction of our prevailing knowledge of field manifestations. The results from this project have brought about a step change in the way we represent heterogeneity in hydraulic properties and how field-scale anisotropy is described. The theory developed in the project has been incorporated into PNNL's STOMP simulator and is currently being used in: 1) tank farm closure studies, 2) design of multilayered surface barriers for closure of U-Plant and BC-Cribs; and 3) fate and transport analysis of the BC Cribs and Trenches, among other off site locations.

The latter application is a particularly good example of the utility of the model. Onedimensional and two-dimensional model simulations based on the existing conceptual model suggested that the $30 \mathrm{Mgal}$ of scavenged tank waste containing an estimated $400 \mathrm{Ci}$ of ${ }^{99} \mathrm{Tc}$ as well as large quantities of $\mathrm{NO}_{3}{ }^{-}$and ${ }^{238} \mathrm{U}$, discharged at $\mathrm{BC}$ Cribs and Trenches would have reached ground water in a matter of weeks of discharge. However, ground water sampling showed no evidence of contaminants. A single borehole installed through the 216-B-26 trench in December 2003 showed contaminants high above the water table. Figure 8 shows the results of a simulation done with STOMP using the new anisotropy model. The model predicted extensive lateral spreading including commingling of the plumes from the seven trenches. The simulated plume spread over a distance of over $300 \mathrm{~m}$ rather than moving down to the water table $(103 \mathrm{~m})$ as expected.

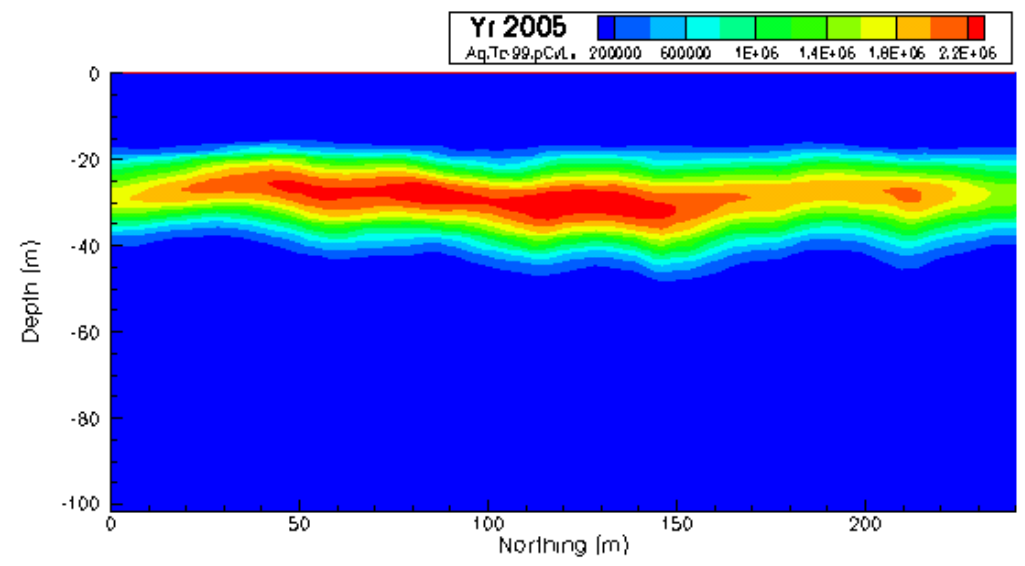

Figure 8. STOMP-Simulated Distributions of Aqueous ${ }^{99}$ Tc in the Year 2005 Based on the new Anisotropy Model for a N-S cross section through Trenches B-52 (N) to B-28 (S) using data from a single bore hole. Correlation structure from a well-characterized site was used to extrapolate parameters to $2 D$.

Figure 9 shows the results of an independent high-resolution-resistivity survey of the site. A more recent survey of the entire site by a private geophysical logging company (hydrogeophysics) shows the plume has spread over a 22-acre region.

\subsection{Planned Activities}

The success of this approach for describing flow in strongly anisotropic sediments and the ability to account for effects of pore-scale heterogeneity over multiple spatial scales provides the ideal platform to study the effects on reactive transport. During the infiltration and redistribution 


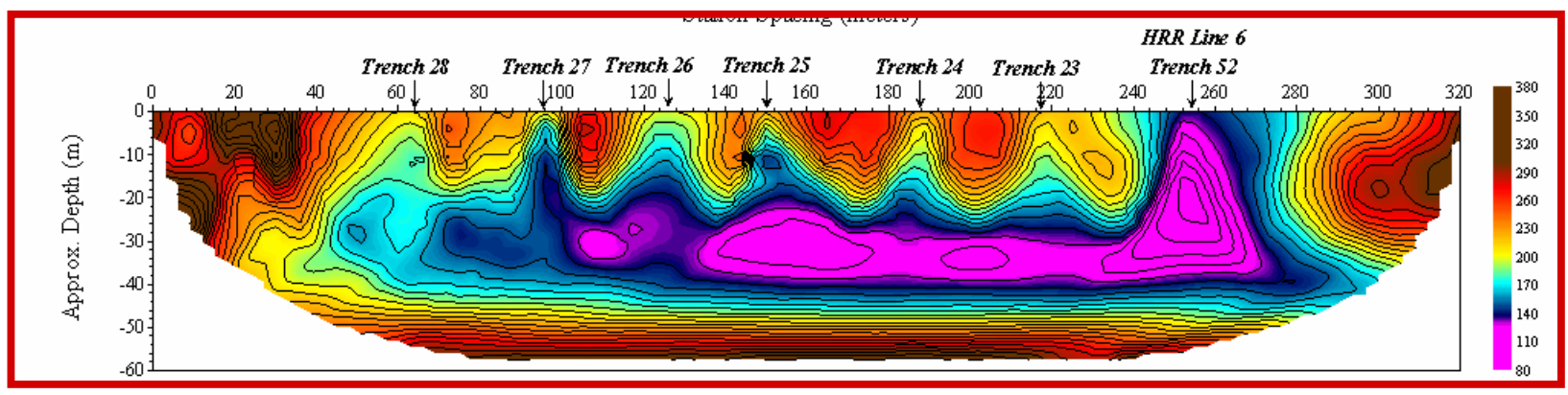

Figure 8. Resitivity Profile in NS Cross-section through Trenches B-52 (N) to B-28 (S) Conducted Independently of STOMP predictions.

of reactive chemicals dissolved in water, access to reactive sites and the kinetics of sorption will be controlled by the pore space that is accessed by the transporting water. The amount of hydrodynamic dispersion is also known to be influenced by the pore-scale heterogeneity. However, very little is known about how these processes interact in the vadose zone to control access to reactive sites and ultimately the extent of adsorption. The next step in this research is therefore to extend these techniques to the study of reactive transport, particularly to the investigation of the dispersivity tensor and its relation to pore morphology and particle size statistics and to the access to reactive sites or sorption. .

\subsection{Information Access}

We are in the process of developing a ling within PNNL's vadose zone web page to highlight the findings of this research. The vadose zone page is located at http://vadose.pnl.gov/.

A list of publications include:

Raats P.A.C., Z.F. Zhang, A.L. Ward, and G.W. Gee. 2004. The relative connectivity-tortuosity tensor for conduction of water in anisotropic unsaturated soils. Vadose Zone Journal 3:14711478.

Stewart, M.L., A.L. Ward, and Rector, D.R. 2005. Lattice-Boltzmann modeling of pore geometry and heterogeneity effects on anisotropy in permeability. Advances in Water Resour. Res.( In Press)

Ward, A.L. 2005. On the Tensorial Nature of Pore Connectivity. (In Review)

Ward, A.L., Z.F. Zhang, G.W. Gee, M.D. White. 2005. Upscaling unsaturated hydraulic parameters for flow through heterogeneous anisotropic sediments: A comparison of methods Advances in Water Resour. Res.(In Press)

Zhang, Z.F., A.L. Ward, and G.W. Gee. 2003. Describing the unsaturated hydraulic properties of anisotropic soils using a tensorial connectivity-tortuosity (TCT) concept. Vadose Zone Journal, 2:313-321. 
Zhang, Z.F., A.L. Ward, and G.W. Gee. 2004. A combined parameter scaling and inverse technique to upscale the unsaturated hydraulic parameters for heterogeneous soils. Water Resour. Res. 40(8), W08306, doi:10.1029/2003WR002925.

Zhang, Z.F., A.L. Ward, and G.W. Gee. 2004. A parameter scaling concept for estimating fieldscale hydraulic functions of layered soils. Journal of Hydraulic Research, 41(extra):93-103. 